\title{
As técnicas projetivas em estudos sobre a consumer culture theory - CCT
}

\author{
Projective techniques in studies on consumer culture theory - CCT
}

\author{
Gustavo Tomaz de Almeida ${ }^{1}$
}

\begin{abstract}
Resumo
Existe uma diversidade de métodos de pesquisa empregados nos estudos do Marketing, incluindo àqueles que discorrem sobre a Consumer Culture Theory - CCT, oriundos de diversos campos, como a Sociologia, a Antropologia e a Psicologia. $\mathrm{O}$ objetivo deste ensaio teórico é refletir sobre o uso das técnicas projetivas em pesquisas qualitativas nacionaisdo Marketing, em especial contribuições metodológicas à CCT. Para tanto, na fase inicial, discorre-se sobre a trajetória das técnicas projetivas, da Psicologia à Administração, seguindo com a descrição de alguns tipos de testes projetivos e findando com as contribuições dessas aos estudos da CCT. Nesta etapa, para fortalecer os argumentos, apresentam-se trabalhos da Administração que adotaram tais técnicas como instrumento de coleta de dados, bem como se apontam sugestões para novas investigações. Como principais contribuições, observam-se que os testes projetivos por acadêmicos do Marketing são subutilizados, bem como se reúnem proposições para maximizar o seu uso em trabalhos futuros, alinhadas com as discussões contemporâneas da CCT, como a Assemblage Theory.
\end{abstract}

Palavras-chave: Técnicas projetivas; Administração.CCT.

\begin{abstract}
There are a variety of research methods used in marketing studies, including those who talk about Consumer Culture Theory (CCT) from a variety of fields, such as Sociology, Anthropology and Psychology. The objective of this theoretical discussion is to reflect on the use of projective techniques in qualitative national marketing research, especially methodological contributions to CCT. To do so, in the initial phase, discuss the trajectory of Projective Techniques, from Psychology to Administration, followed by the description of some types of projective tests and ending with their contributions to the studies of the CCT. At this stage, to strengthen the arguments, there are works of the Administration that adopted these techniques as instrument of data collection, as well as suggestions for new investigations. As main contributions, it is observed that the projective tests by marketing academicsare underutilized; as well as propositions to maximize their use in future researches, aligned with the contemporary discussions of the CCT, such as Assemblage Theory.
\end{abstract}

Keywords: Projective techniques; Administration. CCT.

\section{Introdução}

A subjetividade de um entrevistado nem sempre é capturada por instrumentos mais tradicionais, o que leva os pesquisadores de Marketing a conhecerem os demais métodos (MAFEZZOLLI et al., 2009). É no intuito de compreender melhor outras abordagens e contribuir para os estudos qualitativos que esse ensaio teórico propõe discutir as técnicas projetivas e sua relação com a consumer culture theory (CCT). Sumariamente, essa técnica busca compreender o valor simbólico e os significados do contexto para o participante da pesquisa, levando-o a projetar mais facilmente alguns aspectos subjetivos que, por algum motivo, não faria espontaneamente. Em termos conceituais, há um consenso na Administração de que as técnicas projetivas equivalem a certas formas não estruturadas e indiretas de questionar os entrevistados, direcionando-os a expressarem suas motivações, crenças, atitudes e sensações subjacentes ao problema

Doutorando em Administração - PUC Minas.Brasil. Afiliação: PUC- Minas. Lattes: http://lattes.cnpq.br/7238792084154808 Email: gustavo. almeida@uemg.br 
de pesquisa; revelandomaneiras particulares de ver, sentir e interpretar os fatos (PITROWSKY, DA COSTA, SALLS, 2014).

No contexto brasileiro, acadêmicos da Psicologia têm usado amplamente as técnicas projetivas para avaliação da psique dos sujeitos, com temas relacionados ao alcoolismo, compulsão alimentar, depressão, esquizofrenia, estresse ocupacional, funções executivas desenvolvidas por idosos, ideação suicida, fatores que levam a irritabilidade e agressividade, organização cognitiva de crianças surdas, toxicomania, transtorno de identidade, transtorno do pânico, dentre outros temas (MIGUEL, 2014). Essa difusão no campo da Psicologia leva a problematizar e pressupor se as pesquisas da Administração não estariam subutilizando as técnicas projetivas. Isto porque, ao longo da discussão desse ensaio teórico, é possível notar que a frequência do uso das técnicas projetivas nas pesquisas da Administração, incluindo o Marketing, é relativamente pequena. Por exemplo, no período de 2006 até fevereiro de 2018, há apenas treze artigos publicados nos eventos da ANPAD, sendo apenas nove relacionados ao consumo.

Em periódicos, o resultado não é diferente. Por exemplo, ao analisar os trabalhos publicados na Revista Ciências Administrativas - RCA de 1989 (primeira edição do periódico) até fevereiro de 2018, apenas dois estudos aplicaram as técnicas projetivas. No caso do primeiro, para analisar os estilos de vida dos consumidores do canal de venda direta que compram por meio de catálogo (MODANEZ et al., 2009). Já o segundobuscou compreender o sentido do consumo para as gerações X, Y e Z(ALMEIDA, ITUASSU, MOURA, 2016). Em ambos, os testes projetivos visaram deixar os entrevistados mais à vontade, tentando acessar aquilo que uma pergunta direta talvez não permitisse.

No tocante a Consumer Culture Theory - CCT, que também é foco deste ensaio, vale esclarecer que essa marca foi cunhada em 2005, em uma publicação no Journal of Consumer Research - JCR (ARNOULD, THOMPSON, 2015), e reúne trabalhos de orientação cultural produzidos desde a década de 1980 , anteriormente categorizados como estudos interpretativos ou pós-modernos. Seu foco é revelar as relações dinâmicas entre consumo, mercados e cultura. Na atualidade, o termo é amplamente legitimado pela comunidade acadêmica em diferentes países e, ao mesmo tempo, a marca CCT legitima e aproxima a própria comunidade de pesquisadores.

Frente a essas questões, este estudo teve como questão de pesquisa: De que forma o uso das técnicas projetivas pode contribuir, ainda mais, para as pesquisas qualitativas nacionais do Marketing, em especial aumentando as possibilidades metodológicas da Consumer Culture Theory - CCT?

Ajustificativa de se propor este ensaio teórico está nos novos insights que podem surgir por intermédio de sua leitura para os pesquisadores de Marketing. Espera-se que se conheçam alguns cuidados e oportunidades futuras para se trabalhar com as técnicas projetivas, já que sua utilização "[...] em marketing deve ser feita com bastante cuidado". Em outras palavras, a forma como o pesquisador conduz a pesquisa quepode levar a resultados distorcidos, se ele não se atentar aos procedimentos de validade e confiabilidade dos testes projetivos (MARCHETTI, 1995, p. 21). Em sintonia, Vieira e Tibola (2005) lembram que o pesquisador deve conhecer a teoria o suficiente para inserir as técnicas projetivas em entrevistas. Para tanto, é preciso saber quais tipos são os mais adequados para cada contexto, entre fotos, objetos, completar frases, dentre outros. Além disto, Vieira e Tibola (2005) sugerem que a análise de dados envolva uma equipe multidisciplinar, incluindo profissionais antropólogos, psicólogos, sociólogos, terapeutas e outros, para aumentar as chances de compreensão dos dados.

Ainda sobre a relevância deste ensaio, embora existamcríticas ao método, vale desmistificar. Miguel (2014) aponta que o uso desse método tem revelado índices elevados de validade, refutando o pressuposto de que a subjetividade do profissional leva a resultados distorcidos absurdos. Contudo, o acadêmico deve ter um conhecimento sólido para interpretar adequadamente o campo, visto que os testes projetivos não são instrumentos simples, daí a contribuição de propor este ensaio, organizando para a academia o que já se produziu em trabalhos anteriores, bem como as potencialidades para novas pesquisas.

Finalmente, em termos metodológicos, o trabalho foi organizadoa partir da revisão de literatura, do tipo narrativa, tendo como etapa principal o levantamento bibliográfico. Nesta fase, por meio do mapeamento, iniciou-se a revisão sobre a origem das técnicas projetivas e sua evolução até os dias atuais, partindo da Psicologia, mais especificamente da Psicanálise, até o uso na Administração. Adiante, revisaram-seos 
diversos tipos de técnicas projetivas, categorizadas em quatro: a) Associação, b) Conclusão, c) Construção e d) Expressivas. Ao final, a revisão de literatura permitiu apresentar as contribuições do uso das técnicas projetivas para aCCT a partir daanálise de trabalhos dos eventos da ANPADcujo trabalho completo estivesse disponível em sua página eletrônicaaté fevereiro de 2018. Além disso, revisaram-se os trabalhos em periódicos da Administração publicados até fevereiro de 2018 e indexados no SPELL, tendo encontrado o primeiro em 1997.

A busca nessas duas frentes se deu pela relação direta com a Administração. O critério de seleção dos artigos ocorreu pela procura dos termos projetiva, projetivo e projeção nos campos título, palavras-chave e resumo. Havendo dúvida sobre o emprego dos métodos projetivos, foi feita a leitura da metodologia do trabalho e, caso confirmado, o trabalho completo foi lido. Foram excluídos os artigos construídos em outras áreas (como Contabilidade e Turismo) ou que fugiam do escopo (por exemplo: projeção de resultados contábeis). A revisão é encerrada com as reflexões, limitações e potencialidade para futuros trabalhos, convidando demais pesquisadores aaprofundarem ainda mais em novas interlocuções.

\section{As técnicas projetivas}

\subsection{Breve evolução histórica}

O termo denominado técnicas projetivasteve origem no século XIX, em 1939, no trabalho de Frank denominado Métodos Projetivos para o Estudo da Personalidade, no qual indicava que elas possibilitavam uma compreensão global e dinâmica do paciente. Empiricamente, os primeiros estudos que fizeram uso dessas técnicas estavam no campo das ciências comportamentais, e apareceram entre 1940 e 1960, proliferandose nas áreas da Psicologia clínica e do desenvolvimento, bem como na Sociologia e Antropologia (ROOK, 2006). Nesse período, o termo surge em uma carta de Freud sobre a paranoia, evocando a situação do sujeito se projetar no mundo externo (FREUD, 2006), e passa por outras obras, como Totem e Tabu (FREUD, 2006a), na qual a projeção não se dá apenas com o conflito, mas também como um mecanismo normal que faz parte da percepção de mundo,sendo alterada por vivências afetivas anteriores.

Em umtrabalho, em1953, Skinnner (2003) buscou narrar uma sequência de sílabas, sem sentido inicialmente, nas quais, após uma série de repetições, os pacientes começavam a identificar sons, descrevendo algo cujo sentido poderia ser encontrado em sua história de vida, acessando seu inconsciente e revelando algo que o paciente desconhecia em seu comportamento.

Roudinesco e Plon (1998) compactuam afirmando que o método é originário, de fato, da Psicanálise, mas as demais áreas da Psicologia utilizaram para compreender areação inconsciente por meio da qual o sujeito projeta, num outro ou em um objeto, sentimentos provenientes dele mesmo, mas que ele desconhece ou recusa. Rook (2006) pontua, entretanto, que o método não é exclusivo dessa área, já que pode contribuir, por exemplo, para questões metodológicas do Marketing que avaliem o contexto do consumidor. Esta discussão, se as técnicas podem ou não ser estendidas para outras áreas,é tão presente que Meyer e Kurtz (2006), por exemplo, propuseram substituir o nome por técnicas de desempenho, ou de resposta livre, ou implícitas, ou ainda expressivas, a fim de evitar a má interpretação do seu uso. Contudo, na prática, essa mudança não ocorreu e o termo continuou sendo reconhecido como técnicas projetivas.

Atrelando, agora, com o consumo, segundo Rook (2006), o uso dessas técnicas se difundiu no desenvolvimento de inúmeros estudos após a Segunda Guerra Mundial. Ainda de acordo com o autor, os primeiros estudos que fizeram essa aliança remontam da década de 1950, como Rogers e Beal (1950) e Smith(1954); e seguiram com a pesquisa de Haire (1958).

Rook (2006) também destaca que, na década de 1970,sua popularidade diminuiu drasticamente, perdendo campo para os grupos focais, principalmente pela propagação das análises controladas por computadores. Nos anos de 1980, são poucos os livros que citam esse método, inclusive noMarketing. Para o autor, o declínio só não foi tão acentuado no Reino Unido e na Europa, devido ao desenvolvimento da pesquisa em diferentes paradigmas e ao uso constante da indagação qualitativa em investigações sobre consumo. 
Só na década de 1990 que os Estados Unidos retomaram o interesse por esse instrumento, principalmente em entrevistas individuais. Estudos dos anos de $2000 \mathrm{em}$ diante apontam que tanto as pesquisas sobre comportamento do consumidor quanto os estudos em Marketing têm seções que justificam o uso de técnicas projetivas em várias situações e nos diferentes paradigmas epistemológicos (ROOK, 2006). Pesquisas ainda mais recentes incentivam seu uso no campo do Administração, considerando um método de avaliação que permite aos indivíduos organizarem suas experiências por meio de objetos e materiais, podendo projetar sua personalidade em termos de significados, padrões e sentimentos (PINTO, FREITAS, MENDES, 2013; FARIA, CARVALHO, 2017).

$\mathrm{Na}$ atualidade, a ideia de que as técnicas projetivas são instrumentos exclusivos da Psicanálise é dita como um mito, assim como o questionamento se há validade nessee método, já que não se tem dúvidas sobre a cientificidade deseu uso em diversos contextos, como afirma Miguel (2014). Contudo, o autor também lembra que é ingênuo o pesquisador acreditar ter acesso aos segredos mais íntimos das pessoas, ou que as técnicas projetivas são capazes de descrever um sujeito em sua plenitude.Afinal, todo método apresenta limitações.

\subsection{Conhecendo alguns tipos de técnicas projetivas}

A literatura da pesquisa qualitativa em Administração acena que o uso de um ou mais tipos de técnicasprojetivas é recomendável quando: 1) a informação desejada não pode ser obtida com precisão por métodos diretos; 2) em pesquisas exploratórias, para entendimento e compreensão iniciais e, 3) se o pesquisador tiver um nível de conhecimento aprofundado de suas diretrizes, já que o uso incoerente dos pressupostos das técnicas projetivas pode gerar resultados que não refletem os sujeitos entrevistados (VIEIRA, TIBOLA, 2005).

Para esse debate, o quadro 1 ilustra algumas das técnicas projetivas encontradas na literatura. Utilizam-se exemplos relacionados ao consumo para facilitar o entendimento (Quadro 1).

Quadro 1 - Exemplos de técnicas projetivas

\begin{tabular}{|l|l|l|}
\hline \multicolumn{1}{|c|}{ Técnica } & \multicolumn{1}{|c|}{ Detalhamento } & \multicolumn{1}{c|}{ Exemplo: } \\
\hline $\begin{array}{l}\text { Associação de } \\
\text { palavras ou } \\
\text { figuras }\end{array}$ & $\begin{array}{l}\text { O entrevistado é solicitado a combinar imagens, } \\
\text { experiências, emoções, produtos e serviços, até } \\
\text { mesmo pessoas e lugares, ao objeto de estudo. }\end{array}$ & $\begin{array}{l}\text { Diga o que vem à sua mente quando } \\
\text { pensa na palavra consumo. }\end{array}$ \\
\hline Completar frases & $\begin{array}{l}\text { O entrevistado é solicitado a completar uma } \\
\text { frase. }\end{array}$ & $\begin{array}{l}\text { Se uma pessoa consumisse apenas o } \\
\text { necessário para viver [biologicamente], } \\
\text { o mundo então seria... }\end{array}$ \\
\hline $\begin{array}{l}\text { Elaboração de } \\
\text { desenho }\end{array}$ & $\begin{array}{l}\text { Pede-se ao entrevistado que faça uma figura e e } \\
\text { solicita que ele descreva o sentido dela. }\end{array}$ & $\begin{array}{l}\text { Desenhe algo que representa o que o } \\
\text { consumo significa. }\end{array}$ \\
\hline
\end{tabular}

Fonte: Adaptado de Cooper e Schindler (2011, p.175-176)

Sobre a associação de palavras, descrita no quadro 1, trata-seda técnica projetiva mais antiga, tendo sido utilizada no estudo de Houghton, de 1936. Por sua vez, as técnicas de completar frases têm origemna pesquisa social, de 1953; ao passo que o desenho de figuras aparece inicialmente na pesquisa de Krugman, de 1960 (ROOK, 2006).

Dentro dessas técnicas, o desenho e as explicações atribuídas a ele podem assumir o papel de projetar sentidos, muitas vezes ocultos na fala consciente do momento da entrevista. Apesar do seu potencial ser bastante amplo, permitindo interpretações psicológicas mais aprofundadas, no estudo realizado por acadêmicos da Administração sobre a CCT, o pesquisador deve ter cautela se não houver o envolvimento de profissionais de outras áreas. Isto porque ele deve buscar desenvolver uma análise de conteúdo ou de discurso, por exemplo, da explicação que o próprio entrevistado dá sobre sua representação social do consumo, envolvendo as palavras relacionadas com um material projetivo (por exemplo, o desenho), não recorrendo à Psicanálise para a avaliação do que foi desenhado e descrito (GRASSELI; SOUKI, 2007). Enquanto administrador, ele não tem especialização suficiente para se aventurar na Psicanálise se não 
tiver também a formação exigida nessa área. Por este motivo, a interpretação das técnicas projetivas não necessariamente precisa ser realizada com o apoio de um psicólogo, depende se o foco for a análise de conteúdo ou de discurso da fala, desde que os relatos da pesquisa não avaliem a psique do consumidor.

Ainda sobre seus tipos, as técnicas projetivas costumam ser divididas na literatura deAdministração e Marketing em quatro categorias: a) Técnicas de Associação, b) Conclusão, c) Construção e d) Técnicas Expressivas.

Segundo aponta Cooper e Schindler (2011), Malhotra (2001) e Mattar e Motta (2014), a primeira delas é a mais conhecida e consiste em apresentar ao indivíduo uma lista de palavras ou imagens, pedindo a ele que selecione ou diga a primeira palavra que lhe vemà mente. Esses termos são denominados palavras de teste, pois a lista contém informações neutras ou complementares para disfarçar a finalidade do trabalho. É comum ainda cronometrar o tempo, definindo um momento de corte, e se algum participante responder após esse período, isso acende um alerta para o pesquisador buscar compreender quais os motivos que deixaram o consumidor pensativo em relação às possibilidades.

Malhotra (2001) complementa que se pode ainda contar a frequência com que um termo surgiu entre os diferentes entrevistados, a quantidade de hesitação antes de responder e o número de vezes que uma resposta não foi dada. Assim, por exemplo: "quanto mais um indivíduo hesitar antes de responder, maior será o nível presumido de envolvimento com o assunto" (p.134). Uma ausência de resposta pode ser interpretada como um envolvimento emocional alto, de acordo com o contexto, pois essas pessoas talvez se sintam envolvidas demais para responder em curtos espaços de tempo.

Já as técnicas de conclusão são caracterizadas por Cooper e Schindler (2011) e Mattar e Motta (2014) como uma extensão das técnicas de associação, mas com uma riqueza ainda maior em sentimentos e crenças principais dos indivíduos. Por exemplo, na conclusão de uma frase, os sujeitos envolvidos na pesquisa recebem trechos incompletos de estímulo e são levados a completá-los com a primeira ideia que vemàmente. Na conclusão da história, os participantes se inteiram de um trecho da narrativa, só o suficiente para direcionar o foco no objetivo principal do estudo, mas não o suficiente para saber o final. Ele, então, é levado a construir a conclusão da narrativa com suas próprias palavras.

A terceira delas - a técnica de construção -é explicada por Mattar e Motta (2014) como uma resposta do entrevistado a um desenho animado ou quadrinho, sendo um método de coleta ainda menos estruturado, já que cabe mais interpretação do entrevistado e menos participação do pesquisador. Na técnica de respostas para quadrinhos, pede-se ao participante que escreva uma história descritiva, um diálogo ou uma descrição sobre o assunto focal. Por exemplo, na técnica de elicitação por metáfora de Zaltman, que é uma subdivisão da técnica de construção, é solicitado ao pesquisado que traga de 12 a 15 figuras para a entrevista e, descreva o conteúdo de importância de cada uma das imagens. A análise das falas pode revelar os valores, atitudes e crenças que eles possuem.

Nessa linha, na técnica de seleção de fotos, os entrevistados recebem diferentes figuras e selecionam a pessoa que mais chama sua atenção, ou outra foto fora das apresentadas. Neste último caso, antes ou durante a apresentação, o entrevistado é comunicado sobre o contexto da pesquisa desenvolvida. Uma última subdivisão envolve a elaboração de figuras ou desenhos, pedindo ao entrevistado que explique o sentido implícito, para expressar seus sentimentos a respeito da discussão (MATTAR; MOTTA, 2014).

A última divisão, denominada técnicas expressivas, refere-seasituações verbais ou visuais para os entrevistados apresentarem sentimentos e atitudes de um terceiro. As mais usuais são a dramatização e a técnica de terceira pessoa. Segundo Cooper e Schindler (2011), Malhotra (2001) e Mattar e Motta (2014), na dramatização, o pesquisador pede ao entrevistado que assuma o papel ou comportamento de outra pessoa, e o investigador terá o pressuposto de que os entrevistados projetarão seus próprios sentimentos no papel representado. Por sua vez, na técnica de terceira pessoa, o participante deve relacionar crenças e atitudes pessoais de um amigo, vizinho, colega e, novamente, o pressuposto é que, ao projetar-se em uma terceira pessoa, ele esteja demonstrando sentimentos própriosna prática. Em outras palavras, ao pedir que o sujeito fale algo em terceira pessoa, talvez reduza a pressão de ele ofereceruma resposta socialmente aceita (COOPER, SCHINDLER, 2011), mas que não reflita suas percepções. 
Compreendidasas diversas opções de técnicas projetivas, embora não se limitem a essas de acordo com as diversas obras, pode-se agora sintetizar as vantagens e limitações do seu uso (Quadro 2).

Quadro 2 - Vantagens e limitações do uso das técnicas projetivas

\begin{tabular}{|l|l|}
\hline \multicolumn{1}{|c|}{ Vantagens: } & \multicolumn{1}{c|}{ Limitações: } \\
\hline $\begin{array}{l}\text { Prova uma resposta que o indivíduo não daria ou } \\
\text { não poderia dar se soubesse o objetivo do estudo }\end{array}$ & $\begin{array}{l}\text { Dificuldade para se avaliar a validade e a confiabilidade } \\
\text { dos testes }\end{array}$ \\
\hline $\begin{array}{l}\text { Muito proveitosa quando para motivações, crenças } \\
\text { e atitudes que estão agindo no pesquisado no nível } \\
\text { subconsciente }\end{array}$ & $\begin{array}{l}\text { Exige um pesquisador bem treinado e interpretação } \\
\text { qualificada para análise do material, o que, dependendo } \\
\text { do objetivo do estudo, pode envolver profissionais de } \\
\text { outras áreas, o que envolve um custo financeiro. }\end{array}$ \\
\hline $\begin{array}{l}\text { Fornecem razões que podem ser mais adequadas a } \\
\text { um determinado problema, já que as respostas são } \\
\text { mais reduzidas do viés social }\end{array}$ & $\begin{array}{l}\text { Existe um sério risco de uma interpretação tendenciosa } \\
\text { e um comportamento menos frequente do entrevistado } \\
\text { em relação as demais entrevistas, já que pode envolver } \\
\text { desenho, música, vídeo, etc., o que traz desconforto } \\
\text { para alguns pesquisados e/ou pesquisadores. }\end{array}$ \\
\hline
\end{tabular}

Fonte: Adaptado de Vieira e Tibola (2005, p.23)

Tendo avançado na revisão, no próximo capítulo expõem-se as vantagens mais focadas para a Administração e, em especial, para osestudos da CCT que utilizamesse método.

\subsection{Contribuiçõesdo seu uso para o campo da Administração}

A validade das técnicas projetivas é algo que divide opinião nos periódicos, embora essa desconfiança esteja bem mais reduzida nos dias atuais. Assim, em relação à discussão se seus instrumentos são capazes de compreender aquilo que se propõem, há pesquisadores que supervalorizam e outros que têmextrema desconfiança, como revela Villemor-Amaral e Werlang (2008). Os dois extremos revelam problemas para a difusão do seu uso.

No primeiro caso, para além desse método, a confiança extrema pode gerar um uso acrítico e reduzir a atençãoparaoutras informações.Apesar disso, não parece haver sentido para o pesquisador assumir uma posição de desconfiança extrema, já que Mihura et al. (2013) afirmam, em uma meta-análise realizada nas publicações internacionais de 1974 até 2011 que utilizam as técnicas projetivas, que há evidências de validade para uma boa parcela das variáveis do teste,concluindo que a desconfiança de alguns pesquisadores pode advir do preconceito excessivo sem antes conhecerem a fundo suas diretrizes.

Além disto, Rook (2006) considera que, desde sua origem, quando as técnicas projetivas também estavam relacionadas ao tema consumo, elas eram (e continuam)atreladas a entrevistas individuais em muitos casos, o que facilita a triangulação em torno de um tema eoferece uma variedade de tarefas para os respondentes, diminuindo a desconfiança em torno do seu uso.Como contribuição que favorece o uso desse tipo de técnica na Administração, existe uma forte possibilidade de os entrevistados considerarem a entrevista um momento agradável e envolvente, o que propiciaria um ambiente receptivo à coleta desse tipo de informação (ROOK, 2006).

Falando mais especificamente no campo da Consumer Culture Theory - CCT, temática que desperta maior interesse neste ensaio, a utilização desse instrumento pode trazer à tona os significados pessoais relacionados ao consumo, além de auxiliar a identificação de estratégias defensivas que ocultem, ou disfarcem, o sentido atribuído ao objeto de pesquisa pelo indivíduo (SOUZA et al., 2007) numa perspectiva interpretativista. Para tanto, o pesquisador pode trabalhar perguntando ao entrevistado, por exemplo, algo sobre o consumo de um terceiro sujeito, tal como um colega, amigo ou vizinho.

Para Dias (2008), uma contribuição dessa perspectiva é a possibilidade de se evitar respostas desfocadas das reais razões que levam os consumidores aassumirem certas atitudes, especialmente aopensar no consumo, que carrega aspectos sociais do que é, ou não, aceitável. A utilização de situação projetiva, portanto, visa evitar que o participante se sinta pressionado em dar respostas corretas aos questionamentos 
sobre o consumo e que deixem de expressar o que realmente pensam ou sentem - o que poderia ocorrer em um questionário com perguntas diretas.

Hair et al. (2005) compactuam com essa ideia, ressaltando que elas permitem o acesso a questões subjacentes, não estruturadas e, por vezes, disponíveis apenas no inconsciente do consumidor. Elas são apropriadas para a identificação de motivações ocultas, pessoais ou sensíveis do consumo que, de outra forma, seriam reprimidas, ou retidas (BODDY, 2005). Nesse âmbito, ele também concorda que uma pergunta direta poderia gerar respostas racionais ou completamente fora do contexto do mercado.

Para Rook (2006), as técnicas projetivas também possibilitam a obtenção de dados em maior profundidade quando os consumidores têm memória fraca, pouco conhecimento sobre si mesmos, pouca articulação verbal ou grande timidez diante de tópicos sensíveis ao mercado. Para Rook (2006), a incapacidade dos consumidores em articular suas respostas é um problema sério em muitos contextos depesquisa. Ele entende que essas técnicas têm potencial para ir além das explicações sobre o comportamento do consumidor, obtendo dados que refletamos níveis mais profundos da personalidade, da motivação e do sentido atribuído pelos sujeitos aos mais diferentes objetos, pessoas e situações.

Sintetizando, então, o que foi dito até aqui, ao utilizar esses métodos se busca entender como as pessoas transformam e procuram externar suas experiências de consumo, na forma de narrativa, de um fenômeno relacionado e em resposta a incentivos, pois, conferindo sentido, os consumidores projetam parte de si. Ao interpretar o fato por outros meios, indiretamente projetam os seus próprios sentidos (MALHOTRA, 2001). Vale lembrar que o termo sentido, aliado a CCT, assume um caráter mais interpretativista.

O sentido é uma construção social, um empreendimento coletivo, mais precisamente interativo, por meio do qual as pessoas - na dinâmica das relações sociais, historicamente datadas e culturalmente localizadas - constroem os termos a partir dos quais compreendem e lidam com as situações e os fenômenos à sua volta (SPINK e MEDRADO, 2000, p. 41).

Desse modo, espera-se obter aquilo que é construído socialmente, potencializando como se deu essa construção de significados, explorando aquilo que não foi dito, que está implícito ou silenciado, mas produz efeitos de sentido (PINTO, FREITAS e MENDES, 2013). No caso do consumo, a temática está carregada de noções de certo e errado, e a natureza indireta e ambígua de perguntas projetivas pode encorajar os consumidores a expressarem mais honestamente seus sentidos verdadeiros sobre gastar dinheiro, comer e beber, assistir à televisão e comprar carros, por exemplo.

Contudo, esses exemplos são vistos com baixa frequência na seara das publicações em Administração, incluindo o Marketing e a CCT. Como evidência, ao pesquisar os anais dos últimos doze anos dos eventos da Associação Nacional de Pós-Graduação e Pesquisa em Administração (ANPAD), foram encontrados apenas treze trabalhos que utilizaram as técnicas projetivas, conforme se detalha no quadro 3. Optou-se pelos eventos da ANPAD por reunirem um bom volume de trabalhos no campo da Administração.

Quadro 3 - Síntese das pesquisas acadêmicas que empregaram técnicas projetivas, nos eventos da ANPAD, 2006 a fevereiro de 2018:

\begin{tabular}{|c|c|c|l|}
\hline Ano & Autor & Tema & \multicolumn{1}{c|}{ Síntese } \\
\hline 2007 & $\begin{array}{c}\text { Souza et } \\
\text { al. }\end{array}$ & Trabalho & $\begin{array}{l}\text { Estudo qualitativo, fundamentado em uma postura interpretativista e } \\
\text { utilizando uma entrevista semiestruturada. Empregaram as técnicas } \\
\text { projetivas de desenho para investigar as percepções dos trabalhadores da } \\
\text { indústria pirotécnica do município de Santo Antônio do Monte/Minas Gerais, } \\
\text { para identificar os efeitos de um trabalho com alto risco de acidente na } \\
\text { subjetividade dos colaboradores investigados. }\end{array}$ \\
\hline 2007 & $\begin{array}{c}\text { Grasseli e } \\
\text { Souki }\end{array}$ & Trabalho & $\begin{array}{l}\text { Pesquisa qualitativa, desenvolvida por meio de entrevistas e baseada na } \\
\text { análise de conteúdo. Também utilizaram técnicas projetivas de desenho } \\
\text { para identificar a imagem que os diversos stakeholders têm da profissão e } \\
\text { do profissional de arquitetura. }\end{array}$ \\
\hline
\end{tabular}


(Continuação)

Quadro 3 - Síntese das pesquisas acadêmicas que empregaram técnicas projetivas, nos eventos da ANPAD, 2006 a fevereiro de 2018:

\begin{tabular}{|c|c|c|c|}
\hline Ano & Autor & Tema & Síntese \\
\hline 2008 & Vanzellotti & ССТ & $\begin{array}{l}\text { Pesquisa qualitativa, tambémfundamentada emuma postura interpretativista } \\
\text { e utilizando análise de conteúdo e de discurso. Optaram pela técnica de } \\
\text { completar palavras e a associação para avaliar a influência da esperança } \\
\text { no consumo de cosméticos antissinais. }\end{array}$ \\
\hline 2009 & $\begin{array}{l}\text { Ceschim, } \\
\text { Marchetti }\end{array}$ & ССТ & $\begin{array}{l}\text { Com abordagem qualitativa, análise de conteúdo e técnica de associação } \\
\text { de imagens, visou compreender, por meio das entrevistas, a natureza } \\
\text { das características percebidas e dos fatores psicológicos associados ao } \\
\text { comportamento inovador em relação a produtos orgânicos. }\end{array}$ \\
\hline 2009 & $\begin{array}{l}\text { Santos et } \\
\text { al. }\end{array}$ & $\mathrm{CCT}$ & $\begin{array}{l}\text { Abordagem qualitativa, utilizando entrevista semiestruturada e análise de } \\
\text { narrativas. A técnica projetiva foi a escolha de fotos pré-selecionadas pelo } \\
\text { pesquisador. Visou identificar e explorar as fases de lealdade por meio dos } \\
\text { temas sentimentos, intimidade, interdependência e auto conexão, sob a } \\
\text { perspectiva do relacionamento consumidor-marca. }\end{array}$ \\
\hline 2010 & $\begin{array}{l}\text { Oliveira, } \\
\text { Tonelli e } \\
\text { Zambalde }\end{array}$ & Trabalho & $\begin{array}{l}\text { Pesquisa qualitativa, sócio-construcionista. Os dados foram submetidos } \\
\text { à análise de discurso. A técnica projetiva utilizada foi a construção de } \\
\text { desenhos. Visou descrever os sentidos atribuídos à hierarquia e à disciplina } \\
\text { e suas implicações na adoção de uma inovação na Sexta Região da Polícia } \\
\text { Militar de Minas Gerais. }\end{array}$ \\
\hline 2010 & $\begin{array}{l}\text { Fontes, } \\
\text { Borelli e } \\
\text { Casotti }\end{array}$ & CCT & $\begin{array}{l}\text { Desenvolvido por meio da entrevista individual, semiestruturada e utilizando } \\
\text { a técnica projetiva baseada em imagens, buscou contribuir para uma melhor } \\
\text { compreensão sobre o consumidor masculino de produtos e serviços de } \\
\text { beleza, refletindo sobre padrões estéticos e práticas relacionadas à beleza } \\
\text { masculina. }\end{array}$ \\
\hline 2010 & $\begin{array}{l}\text { Hemais, } \\
\text { Casotti }\end{array}$ & CCT & $\begin{array}{l}\text { Por meio de uma pesquisa qualitativa e com o uso da análise projetiva do } \\
\text { tipo histórias temáticas, visou entender a insatisfação de consumidores de } \\
\text { baixa renda. }\end{array}$ \\
\hline 2011 & $\begin{array}{l}\text { Borelli, } \\
\text { Hemais e } \\
\text { Dias }\end{array}$ & СCT & $\begin{array}{l}\text { Utilizando a técnica de descrição de personagem, visou compreender } \\
\text { como os consumidores comuns - ou seja, aqueles que não consideram } \\
\text { os impactos de seu consumo - percebem o consumidor consciente, as } \\
\text { alterações de hábitos de consumo inerentes ao consumo consciente e as } \\
\text { motivações para isto. }\end{array}$ \\
\hline 2012 & Walther & ССТ & $\begin{array}{l}\text { Por meio da técnica projetiva da videoelicitação, isto é, gravação de um } \\
\text { vídeo sobre uma temática específica, visou compreender a consumidora } \\
\text { feminina de produtos eróticos, numa postura interpretativista e qualitativa. }\end{array}$ \\
\hline 2013 & $\begin{array}{l}\text { Pádua, } \\
\text { Honório }\end{array}$ & Trabalho & $\begin{array}{l}\text { Buscou comparar a configuração dos vínculos mantidos por professores } \\
\text { com duas instituições mineiras de ensino superior, uma pública e outra } \\
\text { privada. O estudo identificou, utilizando técnica projetiva de associação } \\
\text { - contar história sobre a figura - que os vínculos estão relacionados ao } \\
\text { sentimento de pertencimento, sendo que na instituição pública destacam-se } \\
\text { os termos estabilidade e autonomia, ao passo que, na privada, o destaque } \\
\text { foi para a comunicação existente entre professor-aluno-coordenação. }\end{array}$ \\
\hline 2013 & $\begin{array}{l}\text { Pinto, } \\
\text { Freitas e } \\
\text { Mendes }\end{array}$ & CCT & $\begin{array}{l}\text { Com base na técnica projetiva de elaboração de redação por alunos e por } \\
\text { meio da análise de discurso, buscou investigar o que os jovens entendem } \\
\text { sobre shopping center e mostrar o potencial da articulação das técnicas } \\
\text { projetivas com a análise do discurso para os estudos da CCT. }\end{array}$ \\
\hline 2017 & $\begin{array}{l}\text { Faria e } \\
\text { Carvalho }\end{array}$ & ССТ & $\begin{array}{l}\text { O ensaio teórico buscou investigar a proficuidade das técnicas projetivas } \\
\text { na pesquisa acadêmica do comportamento do consumidor com deficiência } \\
\text { intelectual; indicando } 25 \text { diretrizes para eventuais propostas de investigação } \\
\text { fundadas por tal perspectiva. }\end{array}$ \\
\hline
\end{tabular}

Fonte - Elaborado pelos autores (2018)

Nota-se que, nos últimos dez anos, apenas nove trabalhos aliaram CCT e técnicas projetivas nos eventos da ANPAD, que organiza alguns dos principais encontros dos acadêmicos da Administração no 
Brasil. Além disso, os trabalhos sobre CCT, na sua maioria, são de docentes e discentes do departamento de Administração da Universidade Federal do Rio de Janeiro (UFRJ), sendo frequentes trabalhos dosprofessores Roberta Casotti (COPPEAD-UFRJ), Fernanda Chagas Borelli e Marcus Wilcox Hemais (doutores pelo COPPEAD-UFRJ). Finalmente, isso sugere umpotencial de estudos em outras regiões geográficas do Brasil, que carregam subculturas de consumo específicas. Afinal, não podemos afirmar que um consumidor adolescente do agreste nordestino tenha experiências de consumo idênticas a um jovem de mesma idade no sul do Brasil sem antes efetuarmos estudos nas duas regiões, o que convida outros pesquisadores a realizarem alianças entre a CCT e as técnicas projetivas.

Constatamdo-se a baixa frequência nesses eventos, avançou-se para os periódicos nacionais indexados no SPELL, mas os resultados são similares. Encontraram-se apenas 13 artigos que empregaram as técnicas projetivas, no período de 1997 até fevereiro de 2018, na área da Administração. Vale lembrar que o primeiro artigo indexado no SPELL que utiliza tal técnica foi publicado em 1997,=e e, por isso, esse é o período inicial adotado para análise. No quadro 4,a coluna intitulada periódicoapresenta o Qualis Capes na área de avaliação Administração de Empresas do triênio mais recente (2013-2016).

Quadro 4 - Síntese das pesquisas acadêmicas em Administração que empregaram as técnicas projetivas, publicadas nos periódicos nacionais indexados no SPELL, de 1997 a fevereiro de 2018:

\begin{tabular}{|c|c|c|c|c|}
\hline Ano & Autor & Tema & Periódico & Síntese \\
\hline 1997 & Zanelli & Trabalho & $\mathrm{RAC}-\mathrm{A} 2$ & $\begin{array}{l}\text { O autor utiliza diversos instrumentos de coleta de dados, entre } \\
\text { eles as técnicas projetivas. Contudo, não é mencionada qual a } \\
\text { técnica é utilizada, mas apenas que seu emprego possibilitou } \\
\text { as representações projetivas da integração grupal entre os } \\
\text { empregados. }\end{array}$ \\
\hline 2005 & $\begin{array}{c}\text { Vieira e } \\
\text { Tibola }\end{array}$ & CCT & $\mathrm{RAC}-\mathrm{A} 2$ & $\begin{array}{l}\text { O trabalho menciona vantagens e desvantagens no emprego de } \\
\text { diversos métodos de pesquisa qualitativa utilizada em marketing } \\
\text { para desvendar os pensamentos e as motivações mais subjetivas } \\
\text { do consumidor. A técnica projetiva é um dos métodos, mas é } \\
\text { descrita no artigo de forma objetiva, indicando seu conceito e } \\
\text { quando empregá-la. }\end{array}$ \\
\hline 2008 & $\begin{array}{l}\text { Fonseca, } \\
\text { Gonçalves, } \\
\text { Oliveira e } \\
\text { Tinoco }\end{array}$ & CCT & $\mathrm{RAE}-\mathrm{A} 2$ & $\begin{array}{l}\text { O uso de imagens, associado à construção e a análise de cenários } \\
\text { permitiu compreender que o futuro das comunidades virtuais } \\
\text { (prosumers) é marcado por maior participação e envolvimento } \\
\text { dos usuários, por mais inovações nas possibilidades de interação } \\
\text { e acesso, por maior amplitude de propósitos e por crescente } \\
\text { interferência nas relações sociais e no consumo. }\end{array}$ \\
\hline 2009 & $\begin{array}{l}\text { Modanez } \\
\text { et al. }\end{array}$ & CCT & $\mathrm{RCA}-\mathrm{B} 2$ & $\begin{array}{l}\text { Foram apresentadas imagens de mulheres de diversos estilos } \\
\text { e em lugares diferentes para compreender a segmentação } \\
\text { psicográfica e estilos de vida de consumidores de venda direta } \\
\text { por catálogo. }\end{array}$ \\
\hline 2010 & Alcadipani & Trabalho & $\begin{array}{l}\text { Cadernos } \\
\text { EBAPE - } \\
\quad \text { A2 }\end{array}$ & $\begin{array}{l}\text { A partir de desenhos feitos por trabalhadores durante uma } \\
\text { pesquisa etnográfica, o autor nota forte hostilidade, interpretada } \\
\text { a partir do conceito de violência simbólica de Bourdieu. }\end{array}$ \\
\hline 2012 & $\begin{array}{l}\text { Fontes, } \\
\text { Borelli e } \\
\text { Casotti }\end{array}$ & CCT & READ - B1 & $\begin{array}{l}\text { Desenvolvido por meio da entrevista individual, semiestruturada } \\
\text { e utilizando a técnica projetiva baseada em imagens, buscou } \\
\text { contribuir para uma melhor compreensão sobre o consumidor } \\
\text { masculino de produtos e serviços de beleza, refletindo sobre } \\
\text { padrões estéticos e práticas relacionadas à beleza masculina. }\end{array}$ \\
\hline 2012 & $\begin{array}{l}\text { Beck e } \\
\text { Pereira }\end{array}$ & CCT & GEAS - B2 & $\begin{array}{l}\text { Valeu-se da técnica projetiva de completar sentença, quando o } \\
\text { entrevistado é instruído a completar uma frase de estímulo que } \\
\text { está incompleta com a primeira ideia que lhe ocorra, permitindo } \\
\text { revelar alta preocupação ambiental dos participantes da pesquisa. } \\
\text { Todavia percebeu-se que prevalecem os valores ligados ao } \\
\text { individualismo, à passividade, ao imediatismo e um forte egoísmo } \\
\text { predomina nos comportamentos dos respondentes quando o } \\
\text { assunto é a preocupação ambiental. }\end{array}$ \\
\hline
\end{tabular}


(Continuação)

Quadro 4 - Síntese das pesquisas acadêmicas em Administração que empregaram as técnicas projetivas, publicadas nos periódicos nacionais indexados no SPELL, de 1997 a fevereiro de 2018:

\begin{tabular}{|c|c|c|c|c|}
\hline Ano & Autor & Tema & Periódico & Síntese \\
\hline 2014 & $\begin{array}{c}\text { Pitrowsky, } \\
\text { Costa e Sales }\end{array}$ & Trabalho & $\mathrm{BBR}-\mathrm{A} 2$ & $\begin{array}{l}\text { Por meio da construção de desenhos livres e de } \\
\text { completar frases, o trabalho investigou a relação entre o } \\
\text { imaginário organizacional e a dimensão tácita da criação } \\
\text { do conhecimento, em uma empresa do segmento de } \\
\text { telecomunicações. }\end{array}$ \\
\hline 2014 & $\begin{array}{l}\text { Hemais e } \\
\text { Casotti }\end{array}$ & CCT & $\begin{array}{l}\text { Pensamento } \\
\text { Cont. em } \\
\text { Administração } \\
\text { - B2 }\end{array}$ & $\begin{array}{l}\text { Por meio de uma pesquisa qualitativa e com o uso } \\
\text { da análise projetiva do tipo histórias temáticas, visou } \\
\text { entender a insatisfação de consumidores de baixa renda. }\end{array}$ \\
\hline 2014 & $\begin{array}{l}\text { Paiva; } \\
\text { Santos; } \\
\text { Mendonça e } \\
\text { Melo }\end{array}$ & Ensino & Pretexto - B2 & $\begin{array}{l}\text { Foi empregada a técnica projetiva de associação } \\
\text { de palavras para analisar como se configuram as } \\
\text { competências e e-competências, necessárias e } \\
\text { efetivas, de professores de um curso de graduação em } \\
\text { Administração de um centro universitário. }\end{array}$ \\
\hline 2015 & $\begin{array}{l}\text { Medeiros; } \\
\text { Cruz e Vidor }\end{array}$ & ССТ & $\begin{array}{c}\text { Amazônia, } \\
\text { Organizações e } \\
\text { Sustentabilidade } \\
\text { - B3 } \\
\end{array}$ & $\begin{array}{l}\text { Investigou-se a percepção que consumidores potenciais } \\
\text { possuem frente à oferta de produtos ambientalmente } \\
\text { sustentáveis, através do uso de técnicas projetivas de } \\
\text { complementação de histórias. }\end{array}$ \\
\hline 2016 & $\begin{array}{l}\text { Almeida, } \\
\text { Ituassu e } \\
\text { Moura }\end{array}$ & ССТ & $\mathrm{RCA}-\mathrm{B} 2$ & $\begin{array}{l}\text { Buscou-se identificar o sentido do consumo, entendido } \\
\text { como eixo central da sociedade, além de abranger } \\
\text { raízes e reflexos deste fenômeno. Para tanto, optou-se } \\
\text { por focar os grupos geracionais X, Y e Z. Como método } \\
\text { de coleta de dados, adotaram-se as técnicas projetivas } \\
\text { de associação de palavras, complementação de frases } \\
\text { e elaboração de desenhos. }\end{array}$ \\
\hline 2017 & $\begin{array}{l}\text { Pinto e } \\
\text { Freitas }\end{array}$ & CCT & $\mathrm{O} \& \mathrm{~S}-\mathrm{A} 2$ & $\begin{array}{l}\text { Com base na técnica projetiva de elaboração de redação } \\
\text { por alunos e por meio da análise de discurso, investigou } \\
\text { o que os jovens entendem sobre shopping center e } \\
\text { mostrar o potencial da articulação das técnicas projetivas } \\
\text { com a análise do discurso para os estudos da CCT. }\end{array}$ \\
\hline
\end{tabular}

Fonte - Elaborado pelos autores (2018)

Como se pode notar, apenas 9 trabalhos publicados em periódicos nacionais fizeram uso das técnicas projetivas no contexto da CCT, o que sugere um espaço para maiores interlocuções desse instrumento de coleta de dados no campo da Administração. Buscando contribuir para uma maior utilização dessas técnicas, nos parágrafos a seguir, apontam-se alguns horizontes futuros.

\subsection{Proposições para novos trabalhos}

O primeiro ponto a se destacar para futuras investigações é que raramente se veem novos usos ou abordagens das técnicas projetivas nos estudos anteriores, se restringindo em uma boa parte aos desenhos ou figuras como meio projetivo mais frequente. Assim, trabalhos futuros podem utilizar algumas abordagens mencionadas mais recentemente, como sugerem Pinto e Freitas (2017), que empregaram a elaboração de redações e projetaram um cenário no qual os entrevistados imaginariam ser consumidores em um shopping center. Outra observação para trabalhos futuros está na constatação de que as pesquisas nacionais anteriores geralmente têm feito o uso apenas de uma técnica projetiva, isoladamente. Como observam Almeida, Ituassu e Moura (2016) ao utilizarem 3 possibilidades de técnicas projetivas em seu estudo, o campo foi mais rico, já que conseguiram acessar e triangular informações que talvez não obteriam por meio de uma resposta direta "do tipo por que" ou o uso isolado de uma das técnicas. Isto pelo fatodo tema consumo, por vezes, ser carregado de respostas socialmente aceitas. 
Outra observação é sobre como contornar as críticas que são feitas às técnicas projetivas. Nesse caso, trabalhos futuros podem argumentar que alguns pesquisadores conhecidos no campo da Administração (por exemplo, Alcadipani, 2010; Hemais, 2014; Pinto, 2017 e outros) têm utilizado e recomendado o emprego dessas técnicas, justamente por acessar respostas muito íntimas do participante, que um roteiro semiestruturado dificilmente acessaria. Além disso, boa parte dessas publicações estão em periódicos de Qualis A2 e B1, sugerindo que é um método qualitativo aceito pelos revisores e pelo conselho editorial das revistas. Logo, embora seja preciso avaliar os trabalhos como um todo para compreender sua qualidade, o que se deseja apontar é que o uso das técnicas projetivas tem sido aceito no campo. Em reforço e especificamente sobre o uso das técnicas projetivas no contexto da CCT, um trabalho internacional compactua, afirmando que as técnicas projetivas podem ser empregadas para entender o lado interpretativo do consumidor - como os participantes interpretam seus próprios comportamentos e rituais, bem como os de outras pessoas. Essa aliança entre CCT e técnicas projetivas pode ajudar a evitar formas verbais de autorelato, que talvez sejam tendenciosas por construções cognitivas ou cegas a comportamentos automáticos (MOISANDER, VALTONE, HIRSTO, 2009). Esses autores sugerem o uso das técnicas projetivas não apenas como forma de acessar conteúdos inconscientes individuais, mas com o propósito de conversar sobre pessoas, ou seja, para gerar conversas culturais, não cabendo preconceito quanto a sua aplicação no campo da CCT.

Em termos de temas a serem problematizados e formas de investigação, o recente trabalho de Faria e Carvalho (2017) é uma boa referência, propondo relacionar as técnicas projetivas com os consumidores vulneráveis - no caso do trabalho em questão, os deficientes intelectuais. A despeito dessa possibilidade, uma abordagem relativamente nova e que pode ser aliada a técnicas projetivas é a Transformative Consumer Research - TCR, justamente por ela se preocupar na transformação social dos consumidores vulneráveis. Trata-se de um movimento ou abordagem de pesquisa que tem despertado o interesse de pesquisadores do Marketing ao redor de todo o mundo e atraído publicações em periódicos conhecidos na área. O argumento central da TCR é de que as facetas do mercado modificam positiva e negativamente a percepção de bemestar geral do consumidor (satisfação com a vida, qualidade de vida percebida e felicidade) e/ou o bem-estar dos consumidores - indicadores de bem-estar econômico, social e ambiental -, mas que os pesquisadores de Marketing podem contribuir para promover o bem-estar (MICK et al., 2012).

Contudo, a TCR ainda tem dificuldades para conseguir acessar problemas sociais marcados por traços culturais particulares(MICK et al., 2012). Nesse caso, a técnica projetiva pode ajudar a desvelar discursos socialmente aceitos, já que a TCR trabalha dando escuta a diferentes atores (o consumidor, as organizações privadas, as entidades governamentais, dentre outras). Como exemplos de temas para se trabalhar em pesquisas futuras, Mick et al. (2012) apontam que carecem investigações na perspectiva transformativa para entender melhor as vulnerabilidades do consumidor impulsivo, ou que tenha problemas com o alcoolismo, tabagismo, obesidade, drogas, pobreza, materialismo e alergia de alimento; vulnerabilidade de grupos, como pessoas com deficiência, analfabetos, negros e idosos; e a vulnerabilidade de consumidores de serviços financeiros.

Ainda como contribuição para trabalhos futuros, as técnicas projetivas podem ser úteis para tratar um conceito que vem aparecendo mais fortemente nos últimos anos. Trata-se do termo interseccionalidade, na qual os participantes da pesquisa compartilham, experimentam e gerenciam desvantagens entrelaçadas. Nas pesquisas sobre o consumo, novos trabalhos intersetoriais surgem não só para analisarem as categorias sociais sobrepostas, "mas também fornecem uma análise mais profunda dos processos estruturais que criam e exacerbam a vulnerabilidade do consumidor".

Em complemento, "a interseccionalidade tem uma abordagem [...] ampla e considera os contextos econômicos, sociais, culturais e políticos interligados entre os quais as condições individuais e externas interagem" (SAATCIOGLU, CORUS, 2014, p. 122-123). No caso da proposição do presente ensaio, as técnicas projetivas podem ajudar a compreender os fenômenos relacionados à cultura e ao consumo e suas múltiplas sobreposições de marginalização nos níveis individual e institucional. Nessa situação, as técnicas projetivas, com uma abordagem interseccional, podem facilitar a compreensão contextualizada das experiências dos marginalizados, já que o participante talvez se sinta mais à vontade em não falar em primeira pessoa sobre situações mais complexas. Além disso, a noção de interseccionalidade já prevê o 
emprego de "várias estratégias para explorar as semelhanças e diferenças em todos os grupos sociais que experimentam marginalizações que se cruzam" (SAATCIOGLU, CORUS, 2014, p. 123), sendo uma abordagem aberta para outras possibilidades de coleta de dados.

Adiante, pensando em como o uso das técnicas projetivas tem evoluído e se transformado dentro dos artigos encontrados, o trabalho de Freitas e Pinto (2017) é aquele que mais avança em relação aos anteriores. Enquanto uma parte das pesquisas geralmente aplica a técnica projetiva mencionando-a em algumas linhas da metodologia (com raras exceções, como em Alcadipani (2010) que se preocupou em deixar claro para o leitor o que é a técnica e suas potencialidades ao longo da revisão de literatura), Freitas e Pinto (2017) avançam ao propor teoricamente uma aliança entre a técnica projetiva com a análise do discurso. Nesse caso, as investigações futuras podem trabalhar, por exemplo, com a Análise Crítica do Discurso - ACD, proposta por Fairclough. Essa corrente se distingue das demais escolas, pois se caracteriza essencialmente por possuir uma visão particular da relação que se estabelece entre a linguagem e a sociedade, preocupando-se, principalmente,em revelar os aspectos acinzentados e encobertos das desigualdades sociais. Logo, tanto a ACD quanto as técnicas projetivas têm em comum o fato de trazer à tona esses aspectos encobertos em um discurso socialmente aceito, encarando tal discurso como "uma prática, não apenas de representação do mundo, mas de significação do mundo, constituindo e construindo o mundo em significado"(FAIRCLOUGH, 1992, p. 91).

Por fim, novos trabalhos podem aliar as técnicas projetivas e as novas perspectivas que estão surgindo na CCT, como Assemblage Theory, que engloba conceitos extraídos da teoria ator-rede, teoria da prática e estudos de governamentalidade (ARNOULD, THOMPSON, 2015). Coerente com os pressupostos da Assemblage Theory, as técnicas projetivas podem permitir conceber elementos heterogêneos coexistindo sem realmente formar um todo coerente, buscando por meio de projeções revelar aspectos nem sempre perceptíveis nos campos sociais, que têm histórias particulares de práticas estabelecidas e relações de poder institucionalizadas. Por exemplo, vê-se isso em Barnhart e Peñaloza (2013), ao utilizarem a assemblage para compreender a pluralidade de identidades entre idosos, por vezes acizentadas em discursos totalitários. Os pesquisadores da CCT, empregando teoria assemblage e técnicas projetivas, podem ir além da lógica de hibridação globalizada ("todo mundo junto e misturado") para compreender a cultura e consumo dentro de uma perspectiva de significados heterogêneos.

Concluindo, como oportunidade de pesquisas futuras alinhadas a essa perspectiva, Arnould e Thompson (2015) descrevem exemplos, tais como: de que forma o consumo se manifesta no contexto de identidades familiares, terceirização das responsabilidades parentais, negociação de identidades entre idosos, as comunidades de consumo, imagens de publicidade, evolução dos significados da marca, as práticas de consumo sustentável, dentre outras.

\section{Conclusão}

Este trabalho se propôs a refletir sobre o uso das técnicas projetivas em pesquisas qualitativas e nacionais do Marketing, em especial suas contribuições metodológicas para a CCT. Nesta tarefa, foi possível compreender que parece bastante promissor aliar as técnicas projetivas e o consumo, já que a natureza indireta e ambígua de perguntas projetivas pode encorajar os entrevistados a se desviarem do padrão socialmente esperado, expressando mais honestamente seus sentidos verdadeiros. Frente ao que foi exposto e considerando os poucos estudos nacionais que utilizem as técnicas projetivas, abre-se uma oportunidade para acadêmicos nacionais mergulharem nos aspectos culturais, sociais, históricos e situacionais dos consumidoresfazendo uso de tais instrumentos em sua coleta de dados.

Como limitações, o uso das técnicas projetivas de forma incorreta pode levar a respostas com baixa validade científica. Além disso, se o objetivo for fazer uma análise da psique do consumidor, é fundamental que a pesquisa envolva profissionais de outras áreas, como a Psicologia, já que o Administrador não tem habilitação suficiente para essa tarefa,porque, nessa situação, as técnicas projetivas representariam um teste psicológico, cuja aplicação é competência restrita do profissional de psicologia (FORMIGA, MELLO; 2000; CFP, 2018). 
Nesse sentido, como sugestões para novos trabalhos, fica o convite para mais administradores se aprofundarem na compreensão e aplicação empírica das técnicas projetivas. Pode-se buscar entender outras alianças, além das descritas neste trabalho, propondo uma pesquisa multidisciplinar, que envolva profissionais da Administração, Psicologia, Antropologia, Sociologia e outras áreas, permitindo compreender de forma interpretativa osdiversos consumidores no Brasil, visto a diversidade em um país subdesenvolvido e de territórios tão díspares, o que talvez não ocorra em países com menores extensões territoriais, ou comníveis de desenvolvimento econômico e social maiores. Daí a riqueza e fertilidade do território brasileiro para o campo da Administração, tendo em vista quetécnica projetiva ainda é pouco explorada nestas terras.

\section{REFERÊNCIAS}

ALCADIPANI, R. Violência e masculinidade nas relações de trabalho: imagens do campo em pesquisa etnográfica. Cadernos EBAPE.BR, Rio de Janeiro, v. 8, n. 1, art. 6, p. 92-110, mar. 2010.

ALMEIDA, G. T; ITUASSU, C. T; MOURA, L. R. C. O sentido do consumo para membros das gerações X, Y e Z. Revista Ciências Administrativas - RCA. Fortaleza,v.22, n.2, p. 605-636, jul./dez. 2016.

ARNOULD, E. J.; THOMPSON, C. J. Introduction: Consumer Culture Theory: ten years gone (and beyond). In: THYROFF, A. E.; MURRAY, J. B.; BELK, R. W. (Eds.). Consumer culture theory : Research in Consumer Behavior. Bingley (UK): Emerald Group Publishing Limited, 2015, v.17. p.1-21.

BARNHART, M.; PEÑALOZA, L. Who Are You Calling Old? Negotiating Old Age Identity in the Elderly Consumption Ensemble, Journal of Consumer Research, [S.I], v. 39, p. 1133-1153, Abr. 2013.

BECK, C. G.; PEREIRA, R. C. F. Preocupação ambiental e consumo consciente: os meus, os seus e os nossos interesses. Revista de Gestão Ambiental e Sustentabilidade, São Paulo, v. 1, n. 2, p. 51-78, 2012.

BODDY, C. Projective techniques in market research: valueless subjectivity or insightful reality? A look at the evidence for the usefulness, reliability and validity of projective techniques in market research. International Journal of Market Research, [S.I], v. 47, n.3, p. 239-254, 2005.

BORELLI, F. C.; HEMAIS, M. W.; DIAS, P. I. R. C.. Ecológicos ou Controlados? O Consumidor Consciente sob a Perspectiva do Consumidor Comum. In: ENCONTRO ENANPAD, 35., 2011, Rio de Janeiro - RJ, Anais... Rio de Janeiro: Associação nacional de pós-graduação e pesquisa em administração - ANPAD, 2011, p. 1-15.

BORELLI, F. C.; HEMAIS, M. W.; DIAS, P. I. R. C. Ecológicos ou controlados? Construindo discursivamente o consumidor consciente. Revista Brasileira de Marketing, v. 11, n. 2, p. 18-42, maio/ ago. 2012.

CESCHIM, Gisele Renato; MARCHETTI, Zancan. O Comportamento Inovador entre Consumidores de Produtos Orgânicos: Uma Abordagem Qualitativa. In: ENCONTRO ENANPAD, 33., 2009, Rio de Janeiro - RJ, Anais... Rio de Janeiro: Associação nacional de pós-graduação e pesquisa em administração ANPAD, 2009, p. 1-16.

CFP (Conselho Federal de Psicologia). Perguntas Frequentes. 2018. Disponível em: <http://satepsi.cfp. org.br/faq.cfm> Acesso em: 28 de Fev. 2018.

COOPER, D. R.; SCHINDLER, P. S.. Métodos de Pesquisa em Administração. 10. ed. São Paulo: Bookman Companhia Editora, 2011. p. 762

DIAS, C. A. Grupo focal: técnica de coleta de dados em pesquisas qualitativas. Universidade Federal de Paraíba. 2008. p.1-12. Disponível em: <http://www.ies.ufpb.br/ojs2/index.php/ies/article/ viewFile/330/252>. Acesso em: 07 ago. 2016.

FAIRCLOUGH, N. Discourse and social change. Oxford and Cambridge: Polity, 1992. 
FARIA, M. D.; CARVALHO, J. L. F. C.. A proficuidade das técnicas projetivas na pesquisa do comportamento do consumidor com deficiência intelectual. In: ENCONTRO ENANPAD, 41., 2017, São Paulo - SP, Anais... São Paulo: Associação nacional de pós-graduação e pesquisa em administração ANPAD, 2017, p. 1-16.

FONSECA, M. J.; GONÇALVES, M. A.; OLIVEIRA, M. O. R.; TINOCO, M. A. C. Tendências sobre as comunidades virtuais da perspectiva dos prosumers. RAE-eletrônica, v. 7, n. 2, art. 8, p. 1-27, jul./dez. 2008.

FONTES, O. A.; BORELLI, F. C.; CASOTTI, L. M.. Como ser Homem e Ser Belo? Um Estudo Exploratório sobre Práticas Masculinas de Consumo de Beleza. . In:ENCONTRO ENANPAD, 34., 2010, Rio de Janeiro - RJ, Anais... Rio de Janeiro: Associação nacional de pós-graduação e pesquisa em administração - ANPAD, 2010, p. 1-17.

M. Como ser homem e ser belo? Um estudo exploratório sobre a relação entre masculinidade e o consumo de beleza. REAd. Revista Eletrônica de Administração, Porto Alegre, v. 18, n. 2, p. 400432, maio/ago. 2012.

FORMIGA, N. S.; MELLO, I. Testes psicológicos e técnicas projetivas: uma integração para um desenvolvimento da interação interpretativa indivíduo-psicólogo. Psicol. cienc. prof., Brasília, v. 20, n. 2 , p. 12-19, jun., 2000.

Freud, S.; RASCUNHO, H: Paranoia. Em obras psicológicas completas de Sigmund Freud. Edição standard brasileira. Rio de Janeiro: Imago, 2006, v. 1, p. 253-258.

FREUD, S. Totem e tabu. Em Obras psicológicas completas de Sigmund Freud. Edição standard brasileira. Rio de Janeiro: Imago, 2006a, v. 13. p. 11-163.

GRASSELI, M. F.; SOUKI G. Q. Imagem e Posicionamento Profissional: um estudo exploratório sobre o marketing na arquitetura. In:ENCONTRO ENANPAD, 31., 2007, Rio de Janeiro. Anais... Rio de Janeiro: Associação nacional de pós-graduação e pesquisa em administração - ANPAD, 2007, p. 1-16.

JUNIOR HAIR, J. F.. et al. Análise multivariada de dados. Porto Alegre: Bookman, 2005.

HEMAIS, M. W.; CASOTTI, L. M. Passivos, Reativos e Sentimentais: Consumidores de Baixa Renda Projetam suas Insatisfações. In: ENCONTRO ENANPAD, 34., 2010, Rio de Janeiro. Anais... Rio de Janeiro: Associação nacional de pós-graduação e pesquisa em administração - ANPAD, 2010, p. 1-17. Insatisfações projetadas de consumidores de baixa renda. Revista Pensamento Contemporâneo em Administração, Rio de Janeiro v. 8, n. 4, p. 65-84, out./dez . 2014.

MALHOTRA, Naresh K. Pesquisa de marketing: uma orientação aplicada. 3 ed. São Paulo: Bookman, 2001.

MAFEZZOLLI, E. C. F. et al.. Reflexões sobre o uso de técnicas projetivas na condução de pesquisas qualitativas em marketing. PMKT, [S.I], v.9, p. 37-48, 2009.

MARCHETTI, R. Z. Diversidade e Tendências das Pesquisas Qualitativas de Marketing. Caderno de Ciências Sociais Aplicada, Curitiba-UFPR, p.15-24, 1995.

MEDEIROS, J. F.; CRUZ, C. M. L.; VIDOR, G. Inovação Ambiental Sustentável: atributos esperados e risco percebido na compra de produtos verdes. Amazônia, Organizações e Sustentabilidade, Ananindeua - PA, v. 4, n. 1, p. 109-126, jan./jun. 2015.

MEYER, G. J.; KURTZ, J. E. Advancing personality assessment terminology: time to retire "objective" and "projective" as personality test descriptors. Journal of Personality Assessment,[S.I], v. 87, n.3, 2006, p. 223-225.

MICK, D. G., et al. Transformative consumer research for personal and collective well-being: for personal and collective well-being. New York: Routledge, 2012. 
MIGUEL, F. K.. Mitos e verdades no ensino de técnicas projetivas. Psico-USF. Bragança Paulist, v.19, n.1, p.97-106, jan./abr. 2014.

MIHURA, J. L.; MEYER, G. J.; DUMITRASCU, N.; Bombel, G. The validity of individual Rorschach variables: systematic reviews and meta-analyses of the comprehensive system. Psychological Bulletin, v. 139, n. 3, p.548-605, May, 2013.

MODANEZ, P. S. C. et al. Segmentação psicográfica e estilos de vida de consumidores de venda direta por catálogo. Revista Ciências Administrativas-RCA, Fortaleza, v. 15, n.2, 2009.

MOISANDER, J.; VALTONEN, A.; HIRSTO, H. Personal interviews in cultural consumer research-post structuralist challenges. Consumption, Markets and Culture, v. 12, n. 4, p.329-348. 2009.

OLIVEIRA, B.; MOTTA, S.Pesquisa de marketing: metodologia, planejamento, execução e análise. 7. ed. Rio de Janeiro: Elsevier Academic, 2014, 482p.

OLIVEIRA, F. M.; TONELLI, D. F.; ZAMBALDE, A. L. Explorando a Técnica Projetiva de Construção de Desenhos: Anotações de Uma Experiência de Pesquisa. In: ENCONTRO ENANPAD, 34., 2010, Rio de Janeiro - RJ, Anais... Rio de Janeiro: Associação nacional de pós-graduação e pesquisa em administração - ANPAD, 2010, p. 1-17.

PÁDUA, I. O. P.; HONÓRIO, L. C. Vínculos Organizacionais: comparando professores de instituições mineiras de ensino superior. In: ENCONTRO ENANPAD, 37., 2013, Rio de Janeiro - RJ, Anais... Rio de Janeiro: Associação nacional de pós-graduação e pesquisa em administração - ANPAD, 2013, p. 1-16.

PAIVA, K. C. M. et al. Competências e e-competências de professores de Administração. Revista Pretexto, Belo Horizonte, v. 15, Edição especial, p. 99-115, 2014.

PINTO, M. R.; FREITAS, R. C. Em busca de uma articulação entre técnicas projetivas, análise do discurso e os estudos do consumo. Organizações \& Sociedade, Salvador, v. 24, n. 80, p. 157-176, Jan./Mar. 2017.

PINTO, M. R.; FREITAS, R. C.; MENDES, C. A. F.. Em busca de uma aproximação entre técnicas projetivas, análise do discurso e os estudos de consumo. In: ENCONTRO DE ENSINO E PESQUISA EM ADMINISTRAÇÃO E CONTABILIDADE, 4., 2013, Brasília/DF. Anais... Brasília: Associação nacional de pós-graduação e pesquisa em administração- ANPAD, 2013, p. 1-16.

PITROWSKY, M. C.; COSTA, I. S. A.; SALLES, D. M. R. Imaginário organizacional e dimensão tácita do conhecimento: estudo de caso em empresa do segmento de telecomunicações. Vitória, BBR. v.11, n.5, p. 102-123, set./out. 2014.

ROOK, D. W.Let's pretend: projective methods reconsidered. In: BELK, R. W. (Ed.), Handbook of Qualitative Research Methods in Marketing. Hillsdale, NJ: Edward Elgar Publishing, 2006. Cap. 11, 143-154.

Submetido em: 28/09/17

Aprovado em: 06/03/18 